\title{
A new monoid construction under crossed products
}

\author{
Ahmet Emin ${ }^{1}$, Fırat Ateş ${ }^{1}$, Sebahattin Ikikardeş ${ }^{1 *}$ and Ismail Naci Cangül ${ }^{2}$
}

${ }^{\text {*Correspondence: }}$

skardes@balikesir.edu.tr

'Department of Mathematics,

Faculty of Art and Science, Balikesir

University, Balikesir, Turkey

Full list of author information is

available at the end of the article

\begin{abstract}
In this paper we define a new monoid construction under crossed products for given monoids. We also present a generating set and a relator set for this product. Finally, we give the necessary and sufficient conditions for the regularity of it.
\end{abstract}

MSC: 05C10; 05C12; 05C25; 20E22; 20M05

Keywords: crossed product; semi-direct product; monoid presentation

\section{Introduction and preliminaries}

In [1], some conditions for the regularity of the semi-direct product are given. Moreover, in [2], a new monoid construction under semi-direct product and Schützenberger product for any two monoids is defined, and its regularity is examined. Also, in [3], necessary and sufficient conditions for this new product to be strongly $\pi$-inverse are determined. The regularity and $\pi$-inverse property for the Schützenberger product are studied in [4]. By using similar methods as in these above papers, the purpose of this paper is to define a new monoid construction under a crossed product and to give its regularity.

Definition 1 A crossed system of monoids is a quadruple $(A, B, \alpha, f)$, where $A$ and $B$ are two monoids, and $f: B \times B \rightarrow A$ and $\alpha: B \rightarrow \operatorname{End}(A)$, where $\operatorname{End}(A)$ denotes the collection of endomorphism of $A$, are two maps such that the following conditions hold:

$$
\begin{aligned}
& \alpha_{b_{1}}\left(\alpha_{b_{2}}(a)\right) f\left(b_{1}, b_{2}\right)=f\left(b_{1}, b_{2}\right) \alpha_{b_{1} b_{2}}(a), \\
& f\left(b_{1}, b_{2}\right) f\left(b_{1} b_{2}, b_{3}\right)=\alpha_{b_{1}}\left(f\left(b_{2}, b_{3}\right)\right) f\left(b_{1}, b_{2} b_{3}\right)
\end{aligned}
$$

for all $b_{1}, b_{2}, b_{3} \in B, a \in A$. The crossed system $(A, B, \alpha, f)$ is called normalized if $f\left(1_{B}, 1_{B}\right)=$ $1_{A}$. The map $\alpha: B \rightarrow \operatorname{End}(A)$ is called weak action and $f: B \times B \rightarrow A$ is called an $\alpha$-cocycle.

If $(A, B, \alpha, f)$ is a normalized crossed system, then we have $f\left(1_{B}, b\right)=f\left(b, 1_{B}\right)=1_{A}$ and $\alpha_{1_{B}}(a)=a$ by [5].

Let $A$ and $B$ be monoids, and let $f: B \times B \rightarrow A$ and $\alpha: B \rightarrow \operatorname{End}(A)$ be two maps. Let $A \#_{\alpha}^{f} B:=A \times B$ as a set with a binary operation defined by the formula

$$
\left(a_{1}, b_{1}\right)\left(a_{2}, b_{2}\right):=\left(a_{1}\left(\alpha_{b_{1}}\left(a_{2}\right)\right) f\left(b_{1}, b_{2}\right), b_{1} b_{2}\right)
$$

for all $b_{1}, b_{2} \in B, a_{1}, a_{2} \in A$. Then $\left(A \#_{\alpha}^{f} B, \cdot\right)$ is a monoid with unit $1_{A \#_{\alpha}^{f} B}=\left(1_{A}, 1_{B}\right)$ if and only if $(A, B, \alpha, f)$ is a normalized crossed system. In this case, the monoid $A \#_{\alpha}^{f} B$ is called

(c) 2013 Emin et al.; licensee Springer. This is an Open Access article distributed under the terms of the Creative Commons Attribution License (http://creativecommons.org/licenses/by/2.0), which permits unrestricted use, distribution, and reproduction in any medium, provided the original work is properly cited. 
a crossed product of $A$ and $B$ associated to the crossed system $(A, B, \alpha, f)$ [5]. The reader is referred to [6] and [5] for more details on this material.

Definition 2 Let $A$ and $B$ be monoids. For a subset $P$ of $A \times B$ and $a \in A, b \in B$, we let define $P b=\{(c, d b) ;(c, d) \in P\}$ and $a P=\{(a c, d) ;(c, d) \in P\}$. Then the Schützenberger product of the monoids $A$ and $B$, denoted by $A \diamond B$, is the set $A \times \wp(A \times B) \times B$ (where $\wp(\cdot)$ denotes the power set) with the multiplication given by

$$
\left(a_{1}, P_{1}, b_{1}\right)\left(a_{2}, P_{2}, b_{2}\right)=\left(a_{1} a_{2}, P_{1} b_{2} \cup a_{1} P_{2}, b_{1} b_{2}\right) .
$$

It is known that $A \diamond B$ is a monoid with identity $\left(1_{A}, \emptyset, 1_{B}\right)$, where $\emptyset$ is an empty set (see [7]).

\section{A new monoid construction}

In this section, as one of the main results of the paper, we define a new monoid construction under a crossed product and the Schützenberger product by considering the definitions given in the above section. In order to do that, firstly we give the definition of this new product and then we define its presentation.

Definition 3 Let $A$ and $B$ be monoids. For $P \subseteq A \times B$ and $b \in B$, we define

$$
P b=\{(a, d b) ;(a, d) \in P\} .
$$

Let us consider the following multiplication:

$$
\left(a_{1}, P_{1}, b_{1}\right)\left(a_{2}, P_{2}, b_{2}\right)=\left(a_{1} \alpha_{b_{1}}\left(a_{2}\right) f\left(b_{1}, b_{2}\right), P_{1} b_{2} \cup P_{2}, b_{1} b_{2}\right)
$$

on the set $A \times \wp(A \times B) \times B$, where $f: B \times B \rightarrow A$ and $\alpha: B \rightarrow \operatorname{End}(A)$ are given in Definition 1.

Let us show the associative property:

$$
\begin{aligned}
& \left(\left(a_{1}, P_{1}, b_{1}\right)\left(a_{2}, P_{2}, b_{2}\right)\right)\left(a_{3}, P_{3}, b_{3}\right) \\
& \quad=\left(a_{1} \alpha_{b_{1}}\left(a_{2}\right) f\left(b_{1}, b_{2}\right), P_{1} b_{2} \cup P_{2}, b_{1} b_{2}\right)\left(a_{3}, P_{3}, b_{3}\right) \\
& \quad=\left(a_{1} \alpha_{b_{1}}\left(a_{2}\right) f\left(b_{1}, b_{2}\right) \alpha_{b_{1} b_{2}}\left(a_{3}\right) f\left(b_{1} b_{2}, b_{3}\right),\left(P_{1} b_{2} \cup P_{2}\right) b_{3} \cup P_{3}, b_{1} b_{2} b_{3}\right) \\
& \quad=\left(a_{1} \alpha_{b_{1}}\left(a_{2}\right) \alpha_{b_{1}}\left(\alpha_{b_{2}}\left(a_{3}\right)\right) f\left(b_{1}, b_{2}\right) f\left(b_{1} b_{2}, b_{3}\right), P_{1} b_{2} b_{3} \cup P_{2} b_{3} \cup P_{3}, b_{1} b_{2} b_{3}\right) \\
& \quad=\left(a_{1} \alpha_{b_{1}}\left(a_{2}\right) \alpha_{b_{1}}\left(\alpha_{b_{2}}\left(a_{3}\right)\right) \alpha_{b_{1}}\left(f\left(b_{2}, b_{3}\right)\right) f\left(b_{1}, b_{2} b_{3}\right), P_{1} b_{2} b_{3} \cup P_{2} b_{3} \cup P_{3}, b_{1} b_{2} b_{3}\right)
\end{aligned}
$$

and

$$
\begin{aligned}
\left(a_{1}, P_{1}, b_{1}\right)\left(\left(a_{2}, P_{2}, b_{2}\right)\left(a_{3}, P_{3}, b_{3}\right)\right) \\
\quad=\left(a_{1}, P_{1}, b_{1}\right)\left(a_{2} \alpha_{b_{2}}\left(a_{3}\right) f\left(b_{2}, b_{3}\right), P_{2} b_{3} \cup P_{3}, b_{2} b_{3}\right) \\
\quad=\left(a_{1} \alpha_{b_{1}}\left(a_{2} \alpha_{b_{2}}\left(a_{3}\right) f\left(b_{2}, b_{3}\right)\right) f\left(b_{1}, b_{2} b_{3}\right), P_{1} b_{2} b_{3} \cup P_{2} b_{3} \cup P_{3}, b_{1} b_{2} b_{3}\right) \\
\quad=\left(a_{1} \alpha_{b_{1}}\left(a_{2}\right) \alpha_{b_{1}}\left(\alpha_{b_{2}}\left(a_{3}\right)\right) \alpha_{b_{1}}\left(f\left(b_{2}, b_{3}\right)\right) f\left(b_{1}, b_{2} b_{3}\right), P_{1} b_{2} b_{3} \cup P_{2} b_{3} \cup P_{3}, b_{1} b_{2} b_{3}\right) .
\end{aligned}
$$


Let us denote this new product by $A_{c p} \#_{\alpha}^{f} B$. Then, by the above argument, we say that $A_{c p} \#_{\alpha}^{f} B$ is a monoid with the identity $\left(1_{A}, \emptyset, 1_{B}\right)$.

By the following remark, we explain why this new product is worked on in this paper.

Remark 1 In [8-13] the authors give some new results about the $p$-Cockcroft property of some extensions. So, by using these papers, one can also work on this subject by using this new product. So, one can give some new efficient (equivalently, $p$-Cockcroft) presentation examples. By the way, one can also do further algebraic works on this new product. For instance, in this paper, we give necessary and sufficient conditions for this new product to be regular.

\section{Presentation of $A_{c p} \#_{\alpha}^{f} B$}

Let us consider Remark 1 . In order to do such algebraic work, we need to define the presentation of this new product. So, in the following theorem, we give a presentation of $A_{c p} \#_{\alpha}^{f} B$ as one of the main results of this paper.

Theorem 1 Let us suppose that the monoids $A$ and $B$ are defined by presentations $[X ; R]$ and $[Y ; S]$, respectively. Then $A_{c p} \#_{\alpha}^{f} B$ is defined by generators

$$
Z=X \cup Y \cup\left\{z_{a, b} ; a \in A, b \in B\right\}
$$

and the relations

$R$,

$S=W_{S}$,

$y x=\alpha_{y}(x) y \quad(x \in X, y \in Y)$,

$z_{a, b}^{2}=z_{a, b}, \quad z_{a, b} z_{c, d}=z_{c, d} z_{a, b} \quad(a, c \in A, b, d \in B)$,

$z_{a, b} y=y z_{a, b y}, \quad x z_{a, b}=z_{a, b} x \quad(x \in X, y \in Y, a \in A, b \in B)$,

where $W_{S}$ is the word on $X$.

Proof Let us denote the set of all words in $Z$ by $Z^{*}$. Let

$$
\psi: Z^{*} \longrightarrow A_{c p} \#_{\alpha}^{f} B
$$

be a homomorphism defined by $\psi(x)=\left(x, \emptyset, 1_{B}\right), \psi(y)=\left(1_{A}, \emptyset, y\right)$ and $\psi\left(z_{a, b}\right)=\left(1_{A},\{(a, b)\}\right.$, $\left.1_{B}\right)$, where $x \in X, y \in Y, a \in A$ and $b \in B$. Also, we can easily see that we have

$$
\begin{aligned}
& \left(a_{1}, \emptyset, 1_{B}\right)\left(a_{2}, \emptyset, 1_{B}\right)=\left(a_{1} a_{2}, \emptyset, 1_{B}\right), \\
& \left(1_{A}, \emptyset, b_{1}\right)\left(1_{A}, \emptyset, b_{2}\right)=\left(f\left(b_{1}, b_{2}\right), \emptyset, b_{1} b_{2}\right), \\
& \left(1_{A}, P_{1}, 1_{B}\right)\left(1_{A}, P_{2}, 1_{B}\right)=\left(1_{A}, P_{1} \cup P_{2}, 1_{B}\right), \\
& \left(a, \emptyset, 1_{B}\right)\left(1_{A}, \emptyset, b\right)\left(1_{A}, P, 1_{B}\right)=(a, P, b)
\end{aligned}
$$

for $a, a_{1}, a_{2} \in A, b, b_{1}, b_{2} \in B$ and $P_{1}, P_{2} \subseteq A \times B$. This says that $\psi$ is onto. Now let us show that $A_{c p} \#_{\alpha}^{f} B$ satisfies relations (3)-(7). Let $R=x_{1} x_{2} \cdots x_{s}$, where $x_{1}, x_{2}, \ldots, x_{s} \in X$. Thus the 
relation (3) follows from $\left(x_{1}, \emptyset, 1\right)\left(x_{2}, \emptyset, 1\right) \cdots\left(x_{s}, \emptyset, 1_{B}\right)=\left(R, \emptyset, 1_{B}\right)=\left(1_{A}, \emptyset, 1_{B}\right)$. Also, let $S=$ $y_{1} y_{2} \cdots y_{k}$, where $y_{1}, y_{2}, \ldots, y_{k} \in Y$, then the relation (4) follows from

$$
\left(1_{A}, \emptyset, y_{1}\right)\left(1_{A}, \emptyset, y_{2}\right) \cdots\left(1_{A}, \emptyset, y_{k}\right)=\left(W_{S}, \emptyset, S\right)=\left(W_{S}, \emptyset, 1_{B}\right),
$$

where $W_{S}=f\left(y_{1}, y_{2}\right) f\left(y_{1} y_{2}, y_{3}\right) f\left(y_{1} y_{2} y_{3}, y_{4}\right) \cdots f\left(y_{1} y_{2} \cdots y_{k-1}, y_{k}\right)$. For relations (5), we have

$$
\left(1_{A}, \emptyset, y\right)\left(x, \emptyset, 1_{B}\right)=\left(\alpha_{y}(x) f\left(y, 1_{B}\right), \emptyset, y\right)=\left(\alpha_{y}(x), \emptyset, 1_{B}\right)\left(1_{A}, \emptyset, y\right) .
$$

In fact the relations given in (6) follow from (8), (9) and (10). Now let us show that relations (7) hold by the following:

$$
\begin{aligned}
\left(1_{A},\{(a, b)\}, 1_{B}\right)\left(1_{A}, \emptyset, y\right) & =\left(1_{A},\{(a, b y)\}, y\right) \\
& =\left(1_{A}, \emptyset, y\right)\left(1_{A},\{(a, b y)\}, 1_{B}\right), \\
\left(x, \emptyset, 1_{B}\right)\left(1_{A},\{(a, b)\}, 1_{B}\right) & =\left(x,\{(a, b)\}, 1_{B}\right) \\
& =\left(1_{A},\{(a, b)\}, 1_{B}\right)\left(x, \emptyset, 1_{B}\right) .
\end{aligned}
$$

Thus these above arguments say that $\psi$ induces an epimorphism $\bar{\psi}$ from the monoid defined by (3)-(7), say $M$, onto $A_{c p} \#_{\alpha}^{f} B$.

Let us consider the relations (5) and (7). By using these relations, there exist words $w_{x}$ in $X^{*}, w_{y} \in Y^{*}$ and $w_{a, b} \in\left\{z_{a, b}: a \in A, b \in B\right\}^{*}$ such that $w=w_{x} w_{y} w_{a, b}$ in $M$ for $w \in Z^{*}$. Moreover, it can be noted that relations (6) can be used to prove that there exists a set $P(w) \subseteq A \times B$ such that $w_{a, b}=\prod_{(a, b) \in P(w)} z_{a, b}$. So, we have

$$
\begin{aligned}
\bar{\psi}(w) & =\psi(w)=\psi\left(w_{x} w_{y} w_{a, b}\right)=\psi\left(w_{x}\right) \psi\left(w_{y}\right) \psi\left(w_{a, b}\right) \\
& =\left(w_{x}, \emptyset, 1_{B}\right)\left(1_{A}, \emptyset, w_{y}\right)\left(1_{A}, P(w), 1_{B}\right) \\
& =\left(w_{x}, P(w), w_{y}\right)
\end{aligned}
$$

for any word $w \in Z^{*}$.

Now, let us take $w^{\prime}=w_{x}^{\prime} w_{y}^{\prime} w_{a, b}^{\prime}$ and $w^{\prime \prime}=w_{x}^{\prime \prime} w_{y}^{\prime \prime} w_{a, b}^{\prime \prime}$ for some $w^{\prime}, w^{\prime \prime} \in Z^{*}$. If $\psi\left(w^{\prime}\right)=\psi\left(w^{\prime \prime}\right)$, then, by the equality of these components, we deduce that $w_{x}^{\prime}=w_{x}^{\prime \prime}$ in $A, w_{y}^{\prime}=w_{y}^{\prime \prime}$ in $B$ and $P\left(w^{\prime}\right)=P\left(w^{\prime \prime}\right)$. Relations (3) and (4) imply that $w_{x}^{\prime}=w_{x}^{\prime \prime}$ and $w_{y}^{\prime}=w_{y}^{\prime \prime}$ hold in $M$. So that $w^{\prime}=w^{\prime \prime}$ holds. Thus $\bar{\psi}$ is injective.

\section{Regularity of $A_{c p} \#_{\alpha}^{f} B$}

Let $A$ and $B$ be monoids. As depicted in Remark 1, one can work on this new product to show some algebraic properties. To this end, in this section we define the necessary and sufficient conditions for $A_{c p} \#_{\alpha}^{f} B$ to be regular.

For an element $a$ in a monoid $M$, let us take $a^{-1}$ for the set of inverses of $a$ in $M$, that is, $a^{-1}=\{b \in B: a b a=a$ and $b a b=b\}$. Hence $M$ is regular if and only if, for all $a \in M$, the set $a^{-1}$ is not equal to the empty set.

The reader is referred to [2-4] and [1] for more details.

Let us consider the notations given in Definition 3. Then we have the following theorem as a final main result of this paper. 
Theorem 2 Let $A$ and $B$ be any monoids. The product $A_{c p} \#_{\alpha}^{f} B$ is regular if and only if $A$ is a regular monoid and $B$ is a group.

Proof Let us suppose that $A_{c p} \#_{\alpha}^{f} B$ is regular. Thus, for $\left(a,\left\{\left(1_{A}, 1_{B}\right)\right\}, 1_{B}\right) \in A_{c p} \#_{\alpha}^{f} B$, there exists $(x, P, y)$ such that

$$
\begin{aligned}
& \left(a,\left\{\left(1_{A}, 1_{B}\right)\right\}, 1_{B}\right)=\left(a,\left\{\left(1_{A}, 1_{B}\right)\right\}, 1_{B}\right)(x, P, y)\left(a,\left\{\left(1_{A}, 1_{B}\right)\right\}, 1_{B}\right) \\
& =\left(a x,\left\{\left(1_{A}, 1_{B}\right)\right\} y \cup P, y\right)\left(a,\left\{\left(1_{A}, 1_{B}\right)\right\}, 1_{B}\right) \\
& =\left(a x \alpha_{y}(a),\left\{\left(1_{A}, 1_{B}\right)\right\} y \cup P \cup\left\{\left(1_{A}, 1_{B}\right)\right\}, y\right), \\
& (x, P, y)=(x, P, y)\left(a,\left\{\left(1_{A}, 1_{B}\right)\right\}, 1_{B}\right)(x, P, y) \\
& =\left(x \alpha_{y}(a), P \cup\left\{\left(1_{A}, 1_{B}\right)\right\}, y\right)(x, P, y) \\
& =\left(x \alpha_{y}(a) \alpha_{y}(x) f(y, y), P y \cup\left\{\left(1_{A}, 1_{B}\right)\right\} y \cup P, y^{2}\right) .
\end{aligned}
$$

Thus we have $y=1_{B}$. This gives that $a=a x a$ and $x=x a x$. Hence $A$ is regular. By using the similar argument, for $\left(1_{A},\left\{\left(1_{A}, 1_{B}\right)\right\}, b\right) \in A_{c p} \#_{\alpha}^{f} B$, there exists $(x, P, y)$ such that

$$
\begin{aligned}
& \begin{aligned}
\left(1_{A},\left\{\left(1_{A}, 1_{B}\right)\right\}, b\right) & =\left(1_{A},\left\{\left(1_{A}, 1_{B}\right)\right\}, b\right)(x, P, y)\left(1_{A},\left\{\left(1_{A}, 1_{B}\right)\right\}, b\right) \\
& =\left(\alpha_{b}(x) f(b, y),\left\{\left(1_{A}, 1_{B}\right)\right\} y \cup P, b y\right)\left(1_{A},\left\{\left(1_{A}, 1_{B}\right)\right\}, b\right) \\
& =\left(\alpha_{b}(x) f(b, y) f(b y, b),\left\{\left(1_{A}, 1_{B}\right)\right\} y b \cup P b \cup\left\{\left(1_{A}, 1_{B}\right)\right\}, b y b\right), \\
(x, P, y)= & (x, P, y)\left(1_{A},\left\{\left(1_{A}, 1_{B}\right)\right\}, b\right)(x, P, y) \\
= & \left(x f(y, b), P b \cup\left\{\left(1_{A}, 1_{B}\right)\right\}, y\right)(x, P, y) \\
= & \left(x f(y, b) \alpha_{y b}(x) f(y b, y), P b y \cup\left\{\left(1_{A}, 1_{B}\right)\right\} y \cup P, y b y\right) .
\end{aligned}
\end{aligned}
$$

Here, since we have

$$
\begin{aligned}
& \left\{\left(1_{A}, 1_{B}\right)\right\}=\left\{\left(1_{A}, 1_{B}\right)\right\} y b \cup P b \cup\left\{\left(1_{A}, 1_{B}\right)\right\}, \\
& P=P b y \cup\left\{\left(1_{A}, 1_{B}\right)\right\} y \cup P
\end{aligned}
$$

and, in particular, $\left(1_{A}, y b\right)=\left(1_{A}, 1_{B}\right)$ and $P b y=P$, we get $y b=b y=1_{B}$. This says that $B$ is a group.

Suppose conversely that $A$ is a regular monoid and $B$ is a group. Let us take $(a, P, b) \in$ $A_{c p} \#_{\alpha}^{f} B$. Since $B$ is a group, then there exists $y$ in $B$ such that $b y=y b=1_{B}$. Also, since $A$ is regular, we can take $c=\alpha_{y}(v)$ for some $f(b, y) v \in a^{-1}$ such that $v \in(a f(b, y))^{-1}$. Now, let us consider the following:

$$
\begin{aligned}
a \alpha_{b}(c) f(b, y) \alpha_{b y}(a) f(b y, b) & =a \alpha_{b}(c) f(b, y) a=a \alpha_{b}\left(\alpha_{y}(v)\right) f(b, y) a \\
& =a f(b, y) \alpha_{b y}(v) a=a f(b, y) v a=a, \\
c \alpha_{y}(a) f(y, b) \alpha_{y b}(c) f(y b, y) & =c \alpha_{y}(a) f(y, b) c=\alpha_{y}(v) \alpha_{y}(a) f(y, b) \alpha_{y}(v) \\
& =\alpha_{y}(v) \alpha_{y}(a) \alpha_{y}(f(b, y)) \alpha_{y}(v) \\
& =\alpha_{y}(v a f(b, y) v)=\alpha_{y}(v)=c .
\end{aligned}
$$


Also, by choosing, $P_{2}=P_{1} y \subseteq A \times B$, where $P_{1} \subseteq A \times B$, we get

$$
\begin{aligned}
& P_{1} y b \cup P_{2} b \cup P_{1}=P_{1} \cup P_{1} y b \cup P_{1}=P_{1} \cup P_{1} \cup P_{1}=P_{1}, \\
& P_{2} b y \cup P_{1} y \cup P_{2}=P_{1} y \cup P_{1} y \cup P_{1} y=P_{1} y=P_{2} .
\end{aligned}
$$

Consequently, for every $\left(a, P_{1}, b\right) \in A_{c p} \#_{\alpha}^{f} B$, there exists $\left(c, P_{2}, y\right) \in A_{c p} \#_{\alpha}^{f} B$ such that

$$
\begin{aligned}
\left(a, P_{1}, b\right)\left(c, P_{2}, y\right)\left(a, P_{1}, b\right) & =\left(a \alpha_{b}(c) f(b, y) \alpha_{b y}(a) f(b y, b), P_{1} y b \cup P_{2} b \cup P_{1}, b y b\right) \\
& =\left(a, P_{1}, b\right), \\
\left(c, P_{2}, y\right)\left(a, P_{1}, b\right)\left(c, P_{2}, y\right) & =\left(c \alpha_{y}(a) f(y, b) \alpha_{y b}(c) f(y b, y), P_{2} b y \cup P_{1} y \cup P_{2}, y b y\right) \\
& =\left(c, P_{2}, y\right),
\end{aligned}
$$

where $P_{2}=P_{1} y, b y=y b=1_{B}$ and $c=\alpha_{y}(v)$ for some $f(b, y) v \in a^{-1}$ such that $v \in(a f(b, y))^{-1}$. Hence the result.

\section{Competing interests}

The authors declare that they have no competing interests.

\section{Authors' contributions}

All authors completed the paper together. All authors read and approved the final manuscript.

\section{Author details}

${ }^{1}$ Department of Mathematics, Faculty of Art and Science, Balikesir University, Balikesir, Turkey. ${ }^{2}$ Department of Mathematics, Faculty of Arts and Science, Uludag University, Bursa, Turkey.

\section{Acknowledgements}

Dedicated to Professor Hari M Srivastava.

Received: 12 December 2012 Accepted: 26 April 2013 Published: 15 May 2013

\section{References}

1. Nico, WR: On the regularity of semi-direct products. J. Algebra 80, 29-36 (1983)

2. Ateş, F: Some new monoid and group constructions under semidirect products. Ars Comb. 91, $203-218$ (2009)

3. Karpuz, EG, Çevik, AS: A new example of strongly $\pi$-inverse monoids. Hacet. J. Math. Stat. 40, 461-468 (2011)

4. Ateş, F, Karpuz, EG, Çevik, AS: Regular and $\pi$-inverse monoids under Schutzenberger products. Algebras Groups Geom. 27(4), 455-470 (2010)

5. Agore, AL, Militaru, G: Crossed product of groups applications. Arab. J. Sci. Eng. 33, 1-17 (2008)

6. Agore, AL, Fratila, D: Crossed product of cyclic groups. Czechoslov. Math. J.60(135), 889-901 (2010)

7. Howie, JM, Ruskuc, N: Constructions and presentations for monoids. Commun. Algebra 22(15), 6209-6224 (1994)

8. Ateş, F, Cevik, AS: Separability and efficiency under standard wreath product in terms of Cayley graphs. Rocky Mt. J. Math. 38, 779-800 (2008)

9. Ateş, F, Çevik, AS: Minimal but inefficient presentations for semi-direct products of finite cyclic monoids. In: Proceedings of Groups St Andrews 2005. L.M.S Lecture Note Series, vol. 1, pp. 175-180. Cambridge University Press, Cambridge (2006)

10. Ateş, F, Çevik, AS: The p-Cockcroft property of central extensions of groups II. Monatshefte Math. 150, 181-191 (2007)

11. Cevik, AS: The $p$-Cockcroft property of semi-direct products of monoids. Int. J. Algebra Comput. 13(1), 1-16 (2003)

12. Cevik, AS: Minimal but inefficient presentations of the semi-direct product of some monoids. Semigroup Forum 66(1), 1-17 (2003)

13. Cevik, AS: Efficiency for self semi-direct products of the free Abelian monoid on two generators. Rocky Mt. J. Math. 35(1), 47-59 (2005) 